\title{
Case Report: Could Artemisinin-Based Combination Therapy Prevent Occupational Malaria following Blood Exposure?
}

\author{
Geoffrey Minard, ${ }^{1}$ Sylvie Touche, ${ }^{2} \dagger$ Anne Catherine Delavelle, ${ }^{2} \dagger$ Morgane Bonnet, ${ }^{3}$ Antoine Huguenin, ${ }^{4} \dagger$ and Yohan $N^{\prime}$ Guyen ${ }^{1 *} \dagger$ \\ ${ }^{1}$ Service de Médecine Interne, Maladies Infectieuses et Immunologie Clinique, Hôpital Robert Debré, Centre Hospitalier Universitaire de Reims, \\ Reims, France; ' Service de Médecine et Santé au Travail, Hôpital Maison Blanche, Centre Hospitalier Universitaire de Reims, Reims, France; \\ ${ }^{3}$ Pharmacie Centrale, Hôpital Robert Debré, Centre Hospitalier Universitaire de Reims, Reims, France; ${ }^{4}$ Laboratoire de Parasitologie-Mycologie, \\ Pôle de Biopathologie, Centre Hospitalier Universitaire de Reims, Reims, France
}

\begin{abstract}
Postexposure prophylaxis using artemisinin-based combination therapy (ACT) was prescribed to a malarianaive nurse who experienced an injury with a hollow needle previously used on a patient admitted for severe imported Plasmodium falciparum malaria (blood parasitemia 10.8\%). Artemether-lumefantrine, four tablets twice daily for 3 days, was started 12 hours after exposure, and no side effects were reported. During the six following months, she only developed one episode of fever that was associated with pyelonephritis. Biological follow-up, based on blood smears, molecular biology, and serology, did not evidence $P$. falciparum malaria. This case suggests that ACT can prevent occupational $P$. falciparum malaria following needle-stick injury. We found evidence of only one other unpublished similar case where a Turkish resident doctor did not develop malaria after postexposure prophylaxis using ACT. Such a prophylaxis could be prescribed not only in case of occupational exposure to Plasmodium spp. in nonvector-borne laboratory-acquired infections but also following blood exposure in healthcare setting.
\end{abstract}

\section{INTRODUCTION}

Needle-stick injuries continue to occur in healthcare facilities worldwide, ${ }^{1}$ potentially exposing healthcare workers (HCWs) to blood-borne pathogens (BBPs). The overrepresentation of hepatitis $B(\mathrm{HBV})$, hepatitis $\mathrm{C}(\mathrm{HCV})$, and (HIV) viruses among these BBPs explained the postexposure prophylaxis treatment given to HCWs in this situation. ${ }^{2}$

However, a wide variety of other potential viral, bacterial, and protozoal BBPs has been described. ${ }^{3}$ Among those, the protozoan parasite Plasmodium falciparum has been involved in more than 20 malaria cases after needle-stick injuries in healthcare setting, apart from laboratory-acquired infections. ${ }^{4,5}$ All except one had a favorable outcome once antimalarial treatment had been given in case of overt disease. ${ }^{4}$ Nevertheless, there are almost no published data concerning the efficacy of postexposure prophylaxis in the prevention of malaria after exposure to parasitemic blood in healthcare facilities. Chloroquine has been tried once without success during a laboratory-acquired infection more than 30 years ago. ${ }^{6}$ Since the mid-2000s, artemisinin-based combination therapy (ACT) became the drug of choice for uncomplicated $P$. falciparum malaria because of a higher parasitic clearance rate than other antimalarial drugs. ${ }^{7}$

In the present report, a postexposure prophylaxis using ACT was prescribed to an intensive care unit (ICU) nurse, following needle-stick injury, to prevent malaria occupational transmission.

\section{CASE PRESENTATION}

On November 27, 2019, a 39-year-old Caucasian ICU nurse experienced a deep needle-stick injury with a 21-gauge hollow needle forgotten in a sterile field. This needle had just been used as finder needle during insertion of central venous catheter in internal jugular vein of a 57 -year-old female patient

*Address correspondence to Yohan N'Guyen, Ave. du général Koenig, Reims 51100, France. E-mail: yohan.nguyen@wanadoo.fr †These authors contributed equally to this work. admitted the same day for severe imported $P$. falciparum malaria (blood parasitemia $10.8 \%$ and red blood cells [RBC] 3.5 million/ $\mathrm{mm}^{3}, 1$ day after returning from a 3-week trip to Guinea) (Figure 1A) and before any antimalarial treatment was received by the patient. After immediate wound disinfection by diluted sodium hypochlorite solution, the nurse finished her shift and went to occupational medicine consultation. Blood test confirmed the nurse's immunity against HBV, and no antiretroviral postexposure prophylaxis was prescribed because both the nurse and the patient were HIV (and HCV) seronegative. However, considering the risk of occupational $P$. falciparum malaria, a compassionate ACT (artemetherlumefantrine four tablets twice daily with food, for 3 days) was prescribed to the nurse, who was malaria naive. Hospital pharmacy provided the treatment that she began 12 hours after exposure. No side effects were reported by the nurse. To date, she only developed one episode of fever that was associated with documented pyelonephritis. Biological followup, based on fast thick plus thin blood smears using $5 \mu \mathrm{L}$ of blood and molecular biology, ${ }^{8,9}$ did not evidence $P$. falciparum malaria (Figure 1B and $\mathrm{C}$ ). Serological assays evidenced neither HIV/HCV nor $P$. falciparum antigens' seroconversion (Figure 1C).

\section{DISCUSSION}

Herein, we reported the case of a malaria-naive nurse, who did not develop malaria after ACT postexposure prophylaxis given 12 hours after what we judged to be an at-risk needlestick injury. We could not prove that this favorable outcome was due to ACT postexposure prophylaxis, but we judged it likely.

First, by analogy with other viral BBPs, we presumed the needle-stick injury was here at risk because injury was due to a hollow needle filled with highly parasitemic blood, deep, and occurred in a malaria-naive nurse. Most of the occupational malaria-reported cases occurred after needle-stick injuries both in laboratory and in healthcare setting. ${ }^{4,5}$ There are no data concerning the estimated risk of malaria transmission in 
A

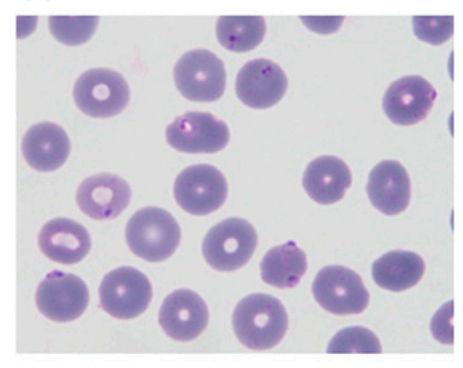

B

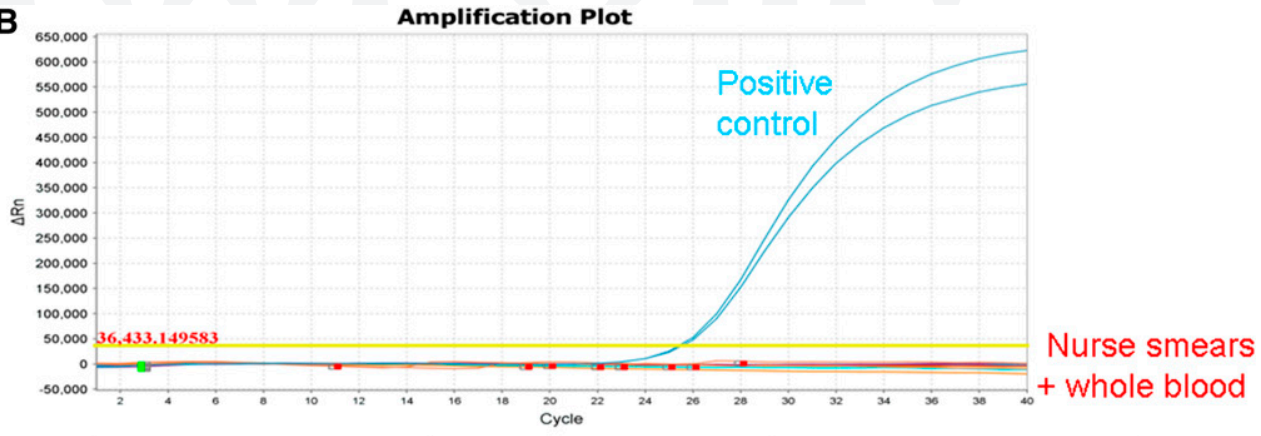

C

\begin{tabular}{|c|c|c|c|c|}
\hline Date & $11 / 28 / 19$ & $12 / 12 / 19$ & $12 / 27 / 19$ & $03 / 02 / 2020$ \\
\hline Blood smears & . & - & - & - \\
\hline PCR & - (smear) & - (smear) & - (smear) & - (smear and whole blood) \\
\hline Malaria Serology & - & $\cdot$ & - & - \\
\hline Haemoglobin $(g / L)$ & ND & 148 & 145 & 141 \\
\hline Platelets (/mm3) & ND & 301 & 309 & 272 \\
\hline ASAT (UI/mL) & ND & 17 & 19 & 20 \\
\hline ALAT (UI/mL) & ND & 16 & 22 & 20 \\
\hline
\end{tabular}

Needlestick injury

Pyelonephritis , ACT

,

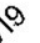

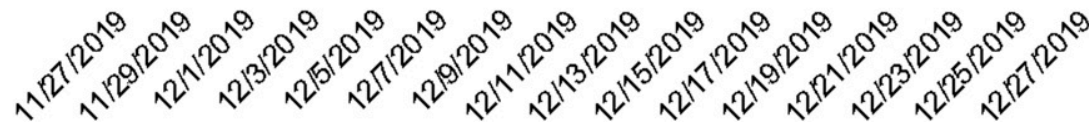

March 2020

FIGURE 1. (A) Thin blood smear of the source, 57-year-old female patient, showing numerous Plasmodium falciparum trophozoites in red blood cells. (B) Amplification curve of pan-plasmodial real-time PCR. In blue, positive control. In red, all curves corresponding to the nurse's samples; in yellow, below threshold detection. PCR has been performed, as previously described, ${ }^{9}$ on the nurse's blood smears and on whole blood during all follow-up and at the end of the 3-month survey, respectively. (C) Results of the biological follow-up performed in the nurse immediately, 2 weeks, and 1 and 3 months after needle-stick injury. Malaria serology has been performed using Malaria EIA Test Kit ${ }^{\circledR}$, Biorad, according to the manufacturer's instructions. Below, time scale with clinical events. ACT = artemisinin-based combination therapy; ND = not done. This figure appears in color at www.ajtmh.org.

these specific situations. The most similar situation where the risk of malaria transmission has been estimated corresponded to transfusion-transmitted malaria. Among Ghanaian patients receiving $\mathrm{RBC}$ transfusion, the transmission rate was only $2-14 \% .{ }^{10}$ Even though the quantity of blood received during RBC transfusion is far superior to that received during a needle-stick injury, the risk of $P$. falciparum malaria transmission after transfusion in Ghana remained low because both blood donors and recipients were immune for malaria, and packed RBC units were parasite free or with very low parasitemia. In our case, nearly 350,000 infected RBCs could be transmitted by $1 \mathrm{~mm}^{3}$ of the patient blood, whereas experimental data suggested that 10 infected RBCs were sufficient to transmit malaria. ${ }^{11}$ Besides, unlike HIV and HCV, the time spent by the needle in the environment did not reduce the risk of transmission of malaria, because all Plasmodium species are able to survive in blood for more than 1 week, even at $4{ }^{\circ} \mathrm{C} .{ }^{11}$

Second, we also presumed that ACT was effective in preventing here likely malaria because there was no evidence for $P$. falciparum malaria in the following months using highly sensitive PCRs, ${ }^{9,10}$ whereas occupational $P$. falciparum malaria usually occurred within a median delay of 12 (range 4-17) days among the 22 cases reported in healthcare setting between 1924 and 2006 without postexposure prophylaxis. ${ }^{4}$ Incubation was similar (median 12.5 days, range 8-14 days) in the five other cases reported up to 2001, without postexposure prophylaxis. ${ }^{5}$ To date, four more cases of occupational malaria have been reported, with a median delay of incubation of 14 (range 9-18) days, all without postexposure prophylaxis. ${ }^{12-15}$ In the sole previously published case postexposure prophylaxis, ${ }^{6}$ $P$. falciparum malaria occurred 17 days after inoculation (breaking a capillary hematocrit tube containing infected blood suspension in laboratory) despite treatment by chloroquine because of acquired resistance of the strain. The potential better efficacy of ACT here is probably due to a higher schizonticidal activity than chloroquine, and such a high schizonticidal activity is probably required after exposure to parasitemic blood, ${ }^{7}$ in which the most abundant parasitic stage corresponded to trophozoites forming in schizonts. In the case of blood-transmitted malaria, infection bypassed the hepatic stage of the parasite life's cycle that is necessary for the activation of innate immunity. ${ }^{5,11}$ This could explain the absence of seroconversion for malarial antigen ${ }^{11}$ we observed here (Figure 1C).

Last, simply reporting this case of absence of $P$. falciparum malaria after ACT postexposure prophylaxis seemed important to us because there is probably no other way (except animal experimental data) to demonstrate a potential benefit of ACT in preventing occupational malaria following blood exposure than gathering evidences issued from real life. To this end, we made exhaustive search and found evidence of only one other similar case, which was unpublished. In that case, a Turkish resident doctor did not develop malaria after taking artemether-lumefantrine as postexposure prophylaxis of $P$. falciparum the day following a deep needle-stick 
injury (while taking a blood sample in a patient treated by artemether-lumefantrine but with $25 \% P$. falciparum blood parasitemia). ${ }^{16}$ This case and ours suggest that ACT can prevent occupational $P$. falciparum malaria following needlestick injury. To gather more evidence, ACT postexposure prophylaxis (which was well tolerated here) could be prescribed not only in case of occupational exposure to Plasmodium spp. in nonvector-borne laboratory-acquired infections but also following blood exposure in healthcare setting. ${ }^{4,5}$ In this latter situation, the parasitemic status of the source patient should be mentioned by the health professional or sought out by the occupational physician because omitting this information could lead to further diagnostic delay and worse outcome. ${ }^{4}$

Received June 22, 2020. Accepted for publication July 27, 2020.

Disclosure: We are indebted to Loren Moufflard and Ailsa Robbins for their help in the preparation of this manuscript.

Authors' addresses: Geoffrey Minard and Yohan N'Guyen, Service de Médecine Interne, Maladies Infectieuses et Immunologie Clinique, Hôpital Robert Debré, Centre Hospitalier Universitaire de Reims, Reims, France, E-mails: XXX and yohan.nguyen@wanadoo.fr. Sylvie Touche and Anne Catherine Delavelle, Service de Médecine et Santé au Travail, Hôpital Maison Blanche, Centre Hospitalier Universitaire de Reims, Reims, France, E-mails: XXX and XXX. Morgane Bonnet, Pharmacie Centrale, Hôpital Robert Debré, Centre Hospitalier Universitaire de Reims, Reims, France, E-mail: XXX. Antoine Huguenin, Laboratoire de Parasitologie-Mycologie, Pôle de Biopathologie, Centre Hospitalier Universitaire de Reims, Reims, France, E-mail: XXX.

\section{REFERENCES}

1. Cheetham S, Ngo H, Liira J, Lee E, Pethrick C, Andrews A, Liira H, 2020. Education and devices to prevent blood and body fluid exposures. Occup Med (Lond) 70: 38-44.

2. Deuffic-Burban S, Delarocque-Astagneau E, Abiteboul D, Bouvet E, Yazdanpanah Y, 2011. Blood-borne viruses in health care workers: prevention and management $J$ Clin Virol 52: 4-10.

3. Tarantola A, Abiteboul D, Rachline A, 2006. Infection risks following accidental exposure to blood or body fluids in health care workers: a review of pathogens transmitted in published cases. Am J Infect Control 34: 367-375.

4. Tarantola A, Rachline A, Konto C, Houzé S, Sabah-Mondan C, Vrillon H, Bouvet E; The Group for the Prevention of Occupational Infections in Health Care Workers, 2005. Occupational Plasmodium falciparum malaria following accidental blood exposure: a case, published reports and considerations for post-exposure prophylaxis. Scand J Infect Dis 37: 131-140.

5. Herwaldt BL, 2001. Laboratory-acquired parasitic infections from accidental exposures. Clin Microbiol Rev 14: 659-688.

6. Jensen JB, Capps TC, Carlin JM, 1981. Clinical drug-resistant falciparum malaria acquired from cultured parasites. Am J Trop Med Hyg 30: 523-525.

7. Lalloo DG, Shingadia D, Bell DJ, Beeching NJ, Whitty CJM, Chiodini PL; PHE Advisory Committee on Malaria Prevention in UK Travellers, 2016. UK malaria treatment guidelines 2016. $J$ Infect 72: 635-649.

8. Thellier M, Datry A, Cissé OA, San C, Biligui S, Silvie O, Danis M, 2002. Diagnosis of malaria using thick bloodsmears: definition and evaluation of a faster protocol with improved readability. Ann Trop Med Parasitol 96: 115-124.

9. Johnston SP, Pieniazek NJ, Xayavong MV, Slemenda SB, Wilkins PP, da Silva AJ, 2006. PCR as a confirmatory technique for laboratory diagnosis of malaria. J Clin Microbiol 44: 1087-1089.

10. Owusu-Ofori AK, Betson M, Parry CM, Stothard JR, Bates I, 2013. Transfusion-transmitted malaria in Ghana. Clin Infect Dis 56: 1735-1741.

11. Verra F, Angheben A, Martello E, Giorli G, Perandin F, Bisoffi Z, 2018. A systematic review of transfusion-transmitted malaria in non-endemic areas. Malar J 17: 36.

12. Miloudi M, Adioui T, Sbaai M, Fatihi J, 2017. Malaria following a blood exposure accident: about a case. Ann Biol Clin (Paris) 75: 455-456.

13. Van Agtmael MA, 1997. A most unfortunate needlestick injury: why the doctor paid a taxi for the nurse. BMJ 314: 337.

14. Vareil M-O et al., 2011. Unusual transmission of Plasmodium falciparum, Bordeaux, France, 2009. Emerg Infect Dis 17: 248-250.

15. Mickiene A, Jonikaite I, Pakalniene J, 2018. Occupationally acquired $P$. falciparum malaria. $J$ Infect Dis Ther 6: 352.

16. Erdinc FS, Yarimoglu S, Aktepe E, Tuncer Ertem G, Tulek N, 2013. Prophylaxis Decision-Making Following Plasmodium falciparum Malaria Needlestick Injury: A Case Report. Poster Presentation 23rd European Congress of Clinical Microbiology and Infectious Diseases (ECCMID), April 27-30, 2013, Berlin, Germany. 